\title{
ТРАЕКТОРИЯ, МИНИМИЗИРУЮЩАЯ ОБЛУЧЕНИЕ ДВИЖУЩЕГОСЯ ОБЪЕКТА
}

\author{
В. И. Бердышев, В. Б. Костоусов
}

В пространстве $X=\mathbb{R}^{N}(N=2,3)$ задан "коридор" $Y$ для движения объекта, вне коридора расположено конечное число излучателей $s_{i}$ с фиксированными выпуклыми конусами излучения $K\left(s_{i}\right)$ и интенсивностью излучения $F(y), y>0$, удовлетворяющей условию $F(y) \geq \lambda F(\lambda y)$ при $y>0, \lambda>1$. В классе траекторий равномерного движения $\mathcal{T}=\left\{t(\tau): 0 \leq \tau \leq 1, t(0)=t_{*}, t(1)=t^{*}\right\} \subset Y, t_{*}, t^{*} \in$ $\partial Y, t_{*} \neq t^{*}$, требуется найти траекторию, минимизирующую величину

$$
J(\mathcal{T})=\sum_{i} \int_{0}^{1} F\left(\left\|s_{i}-t(\tau)\right\|\right) d \tau .
$$

В работе предлагаются способы приближенного построения оптимальных траекторий в случае, когда кратность покрытия "коридора" $Y$ конусами $K\left(s_{i}\right)$ не более двух.

Ключевые слова: навигация, оптимальная траектория, облучение, движущийся объект.

V.I. Berdyshev, V. B. Kostousov. A trajectory minimizing the exposure of a moving object.

A corridor $Y$ for the motion of an object is given in the space $X=\mathbb{R}^{N}(N=2,3)$. A finite number of emitters $s_{i}$ with fixed convex radiation cones $K\left(s_{i}\right)$ are located outside the corridor. The intensity of radiation $F(y), y>0$, satisfies the condition $F(y) \geq \lambda F(\lambda y)$ for $y>0, \lambda>1$. It is required to find a trajectory minimizing the value

$$
J(\mathcal{T})=\sum_{i} \int_{0}^{1} F\left(\left\|s_{i}-t(\tau)\right\|\right) d \tau
$$

in the class of uniform motion trajectories $\mathcal{T}=\left\{t(\tau): 0 \leq \tau \leq 1, t(0)=t_{*}, t(1)=t^{*}\right\} \subset Y, t_{*}, t^{*} \in$ $\partial Y, t_{*} \neq t^{*}$. We propose methods for the approximate construction of optimal trajectories in the case when the multiplicity of covering the corridor $Y$ with the cones $K\left(s_{i}\right)$ is at most 2.

Keywords: navigation, optimal trajectory, irradiation, moving object.

MSC: $00 \mathrm{~A} 05$

DOI: $10.21538 / 0134-4889-2020-26-1-27-38$

\section{1. Введение}

В статье изучается задача построения маршрута, двигаясь с постоянной скоростью по которому объект получит наименьшую суммарную дозу облучения от нескольких источников. Задача рассматривается на плоскости и в пространстве $\mathbb{R}^{3}$. Маршрут должен соединять фиксированные начальную и конечную точки и не выходить за границу заданного коридора $Y$. Граница $\partial Y$ коридора представляет собой замкнутую непрерывную линию в $\mathbb{R}^{2}$ или замкнутую непрерывную поверхность в $\mathbb{R}^{3}$; более точно, анализируется случай, когда граница $\partial Y$ гомеоморфна сфере. Источники расположены вне коридора и являются направленными, т. е. каждый из них излучает в некоторый свой выпуклый конус (конус излучения) с вершиной в точке расположения источника. В работе рассматриваются источники, конусы излучения которых полностью перекрывают коридор $Y$. Считается, что источники являются "однотипными" в том смысле, что они имеют одинаковую зависимость убывания интенсивности излучения от расстояния до источника. В статье исследование ограничено случаем, когда кратность перекрытия коридора $Y$ конусами излучения источников не превышает двух. 
Результаты данной работы могут быть полезны, например, для задач планирования оптимальных путей автономных мобильных роботов в среде с препятствиями [1].

В заданном коридоре $Y \subset \mathbb{R}^{N}(N=2,3)$ движется объект $t$ с постоянной скоростью из начальной точки $t_{*}$ в конечную точку $t^{*}\left(t_{*}, t^{*} \in \partial Y, t_{*} \neq t^{*}\right), \mathbb{T}$ - совокупность траекторий

$$
\mathcal{T}=\left\{t(\tau): 0 \leq \tau \leq 1, t(0)=t_{*}, t(1)=t^{*}\right\} \subset Y .
$$

Граница $\partial Y$ коридора $Y$ гомеоморфна сфере. Вне коридора расположены неподвижные источники излучения $s_{i}(i=1, \ldots, n)$. Излучение осуществляется вдоль лучей $l$ из заданного фиксированного выпуклого открытого конуса $K_{i}=K\left(s_{i}\right)=\{l\}$ с вершиной $s_{i}$, при этом $t_{*} \notin K_{i}, t^{*} \notin K_{i}$. Каждый конус $K\left(s_{i}\right)$ удовлетворяет условию

$$
K\left(s_{i}\right) \cap \mathcal{T} \neq \varnothing \quad \forall \mathcal{T} \in \mathbb{T} .
$$

Через $K_{Y}\left(s_{i}\right)$ обозначим ближайшее к $s_{i}$ связное подмножество из $K\left(s_{i}\right) \cap Y$, удовлетворяющее указанному условию.

Возможны два варианта: граница $\partial Y$ коридора пропускает либо не пропускает излучение. Для определенности будем считать вначале, что граница коридора препятствует распространению излучения. В этом случае через $K_{V}(s)$ будем обозначать множество облучаемых (видимых) из источника $s$ точек множества $K_{Y}(s)$. Интенсивность излучения каждого источника $s=s_{i}$ одинакова вдоль всех лучей из $K\left(s_{i}\right)$ и характеризуется положительной убывающей функцией $\widetilde{F}_{i}(y)$ вещественной переменной $y>0$. Положим

$$
F_{i}(x)=\left\{\begin{array}{cll}
\widetilde{F}_{i}\left(\left\|s_{i}-x\right\|\right) & \text { при } & x \in l \in K\left(s_{i}\right), \\
0 & \text { при } x \notin K\left(s_{i}\right) .
\end{array}\right.
$$

Пусть

$$
J(\mathcal{T})=\sum_{i=1}^{n} \int_{0}^{1} F_{i}(t(\tau)) d \tau
$$

- суммарное облучение, полученное объектом при движении по траектории $\mathcal{T}, n-$ количество излучателей.

В данной работе рассматривается задача построения траектории $\widehat{\mathcal{T}}$, доставляющей точную нижнюю грань

$$
J=\inf \{J(\mathcal{T}): \mathcal{T} \in \mathbb{T}\},
$$

при этом предполагается, что кратность покрытия коридора $Y$ конусами $K\left(s_{i}\right)$ не более двух. Это задача вариационного исчисления с негладкими (если граница $\partial Y$ не является гладкой) ограничениями, усложненная наличием нескольких источников излучения. Очевидно, что возможна постановка задачи (1) как задачи оптимального управления движением объекта при фазовых ограничениях [2]. Здесь предлагаются геометрические способы поиска оптимальной или близкой к оптимальной траектории - в зависимости от взаимного расположения конусов $K\left(s_{i}\right)$. Задача о движении объекта в радиоактивной среде в иной постановке рассматривалась в $[3 ; 4]$.

Естественно предположить, что функция $\widetilde{F}$ не только убывает, но и обладает следующим свойством:

$$
\int_{C_{\rho}} F(x) d x>\int_{C_{\lambda \rho}} F(x) d x \quad \forall \lambda>1,
$$

при любом $\rho>0$, где $C_{\rho}=\{x \in K(s):\|s-x\|=\rho\}$. Это свойство, в частности, имеет функция, для которой выполняется условие

$$
\widetilde{F}(\rho)>\lambda \widetilde{F}(\lambda \rho) \text { при } \rho>0, \lambda>1 .
$$


В самом деле,

$$
\int_{C_{\rho}} F(x) d x=\widetilde{F}(\rho)\left|C_{\rho}\right|>\lambda \widetilde{F}(\lambda \rho)\left|C_{\rho}\right|=\widetilde{F}(\lambda \rho)\left|C_{\lambda \rho}\right|=\int_{C_{\lambda \rho}} F(x) d x,
$$

где $\left|C_{\rho}\right|$ - площадь (длина дуги) $C_{\rho}$.

В случае $\mathbb{R}^{2}$ отметим следующие соотношения для функции $\widetilde{F}$, удовлетворяющей условию (3):

- пусть $[a, b],[\lambda a, \lambda b](\lambda>1)$ - отрезки, лежащие на луче $l \in K(s)$ с вершиной $s=0$, тогда

$$
\int_{a}^{b} F(x) d x>\int_{a}^{b} \lambda F(\lambda x) d x=\int_{a}^{b} F(\lambda x) d \lambda x=\int_{\lambda a}^{\lambda b} F(x) d x
$$

- пусть $\Phi$ - раствор конуса $K=K(s), \Phi<\pi, s=0$ - начало координат полярной системы, $\mathcal{T} \in \mathbb{T}$ и часть этой траектории, $\mathcal{T}_{K}=\mathcal{T} \cap K_{Y}$, задана функцией $\rho=\rho(\varphi)$, и пусть $\lambda \mathcal{T}_{K} \subset K_{Y}$ для $\lambda>1$, тогда

$$
\int_{0}^{\Phi} \widetilde{F}(\rho(\varphi)) d \varphi>\int_{0}^{\Phi} \lambda \widetilde{F}(\lambda \rho(\varphi)) d \varphi>\int_{0}^{\Phi} \widetilde{F}(\lambda \rho(\varphi)) d \varphi .
$$

Таким образом, интеграл $\int_{0}^{\Phi} \widetilde{F}(\lambda \rho(\varphi)) d \varphi$ как функция от $\lambda$ также монотонно убывает с ростом $\lambda$.

Примером функции, удовлетворяющей условию (3), является функция

$$
\widetilde{F}(\rho)=\rho^{-\alpha} \text { при } \alpha \geq 1 .
$$

\section{2. Случай единственного источника излучения $s$ в $\mathbb{R}^{2}$}

Рассмотрим случай единственного источника излучения $s$ на плоскости, тогда

$$
J(\mathcal{T})=\int_{0}^{1} F(t(\tau)) d \tau
$$

Поскольку $t_{*}, t^{*} \in \partial Y$, граница $\partial Y$ разбивается точками $t_{*}, t^{*}$ на две части. Пусть $\Gamma-$ противоположная по отношению к $s$ часть границы коридора $Y$. По кривой $Г$ определим в полярной системе координат с началом $s$ функцию $\rho=\rho_{\Gamma}(\varphi), 0 \leq \varphi \leq \Phi$, так, что величина $\rho_{\Gamma}(\varphi)$ является расстоянием от $s$ до Г по лучу $l_{\varphi} \subset K(s)$, исходящему из $s$ под углом $\varphi$. Функция $\rho_{\Gamma}(\varphi)$ может иметь точки разрыва. Дополним график функции $\rho_{\Gamma}(\varphi)$ в каждой точке разрыва $\varphi$ отрезком $\left[\rho_{\varphi}^{\prime}, \rho_{\varphi}^{\prime \prime}\right], \rho_{\varphi}^{\prime}<\rho_{\varphi}^{\prime \prime}$ луча $l_{\varphi}$ так, чтобы получилась непрерывная кривая, и обозначим ее через gr $\rho(\cdot)$ (см. рис. 1$)$. Заметим, что при движении объекта по полуинтервалу $\left(\rho_{\varphi}^{\prime}, \rho_{\varphi}^{\prime \prime}\right] \subset l_{\varphi}$ объект не облучается, так как точка $\left(\rho_{\varphi}^{\prime}, \varphi\right)$ принадлежит границе $Г$. На первый взгляд, можно ожидать, что $\operatorname{gr} \rho(\cdot)$ и есть оптимальная траектория. Следующие примеры показывают, что кривая $\operatorname{gr} \rho(\cdot)$ не всегда оптимальна.

П р и м е р 1. Пусть $\Gamma$ - график функции $\rho=\rho(\varphi)=1+\varepsilon \sin k \varphi(\varepsilon<1 / 2)$ (см. рис. 2), тогда при достаточно большом $k$ функция $\rho$ имеет большую вариацию $\bigvee_{0}^{\Phi} \rho$ и, значит, большую величину $J(\operatorname{gr} \rho(\cdot))$. Предпочтительней в данном случае траектория, совпадающая на $K(s)$ с дугой окружности с центром $s$ радиуса $1-\varepsilon$.

П р и м е р 2. Для $\rho_{1}<\rho_{2}$, и углов $\varphi_{1}, \varphi_{2}, 0 \leq \varphi_{1}<\varphi_{2} \leq \Phi<\pi$, определим кривую $Г$, состоящую из двух дуг - $\left(\rho_{1}, \varphi\right), 0 \leq \varphi \leq \varphi_{1} ;\left(\rho_{1}, \varphi\right), \varphi_{2} \leq \varphi \leq \Phi,-$ и двух отрезков, 


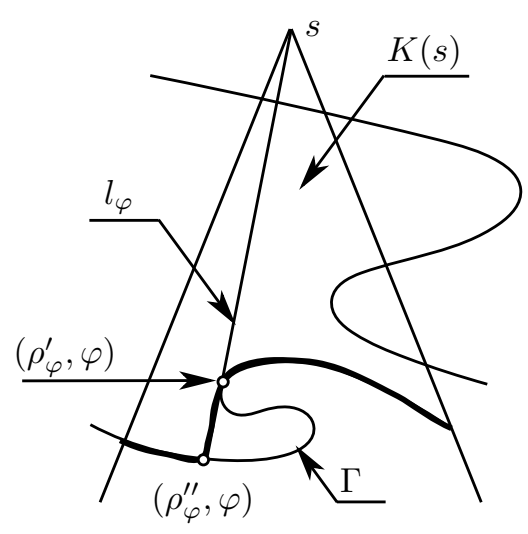

Рис. 1

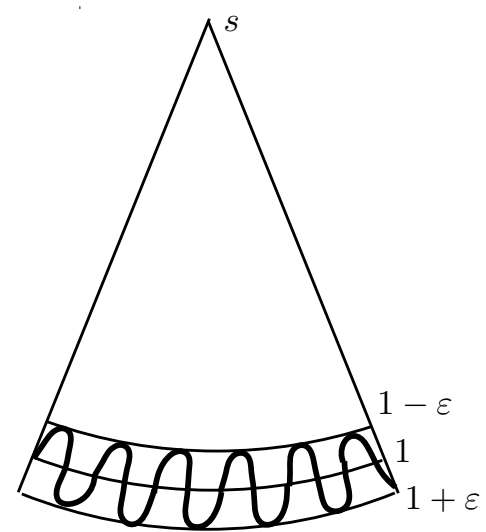

Рис. 2

соединяющих точку $\left(\rho_{2},\left(\varphi_{1}+\varphi_{2}\right) / 2\right)$ с точками $\left(\rho_{1}, \varphi_{1}\right),\left(\rho_{1}, \varphi_{2}\right)$. При большой величине $\rho_{2}-\rho_{1}$ дуга окружности $C_{\rho_{1}}$ более предпочтительна в качестве траектории в сравнении с $\Gamma$.

Данные примеры и свойство монотонности функции $\widetilde{F}$ показывают, что оптимальная траектория должна располагаться по возможности дальше от $s$ и иметь на малых по площади участках малую длину.

Исходная задача является задачей вариационного исчисления поиска непрерывной функции $\rho(\varphi)$ :

$$
\inf _{\rho(\varphi)}\left\{\int_{0}^{\Phi} \widetilde{F}(\rho(\varphi)) d \varphi: \rho(\varphi) \leq \rho_{\Gamma}(\varphi)\right\} .
$$

Трудность этой задачи определяется свойствами функции $\rho_{\Gamma}(\varphi)$.

Предположим, что функция $\rho_{\Gamma}(\varphi)$ кусочно монотонна. Пусть $x=(\bar{\rho}, \bar{\varphi})$ - точка локального максимума функции (см. рис. 3) $\rho_{\Gamma}(\varphi)$; она, возможно, принадлежит отрезку, состоящему из точек максимума, т. е. существуют $\varphi^{\prime} \leq \varphi^{\prime \prime}$ такие, что

$$
\rho_{\Gamma}(\bar{\varphi}) \geq \rho_{\Gamma}(\varphi) \text { при } \varphi^{\prime} \leq \varphi \leq \varphi^{\prime \prime} \quad \text { и } \rho_{\Gamma}(\bar{\varphi})>\min \left\{\rho\left(\varphi^{\prime}\right), \rho\left(\varphi^{\prime \prime}\right)\right\} .
$$

Для $\rho<\bar{\rho}$, близких к $\bar{\rho}$, обозначим через $a=a(\rho)=a\left(\rho, \varphi_{\rho}\right), b=b(\rho)=b\left(\rho, \varphi^{\rho}\right), \varphi_{\rho}<\varphi^{\rho}$, ближайшие к $l_{\bar{\varphi}}$ точки из $C_{\rho} \cap \operatorname{gr} \rho(\cdot)$ и через $\rho^{*}=\rho_{x}^{*}$ - наибольшее из чисел $\rho$, для которых одна

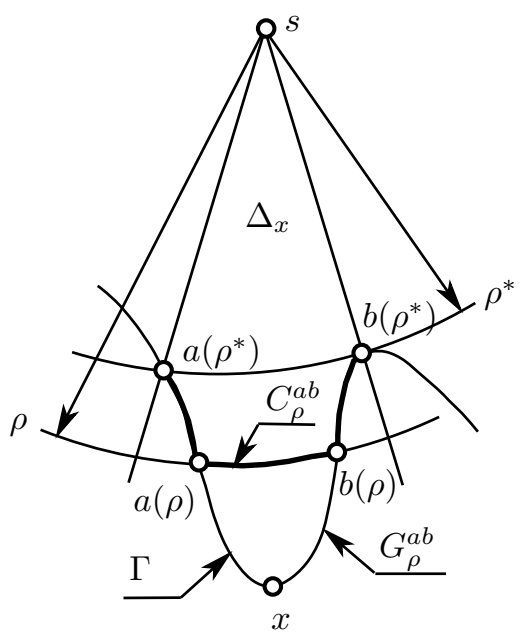

Рис. 3

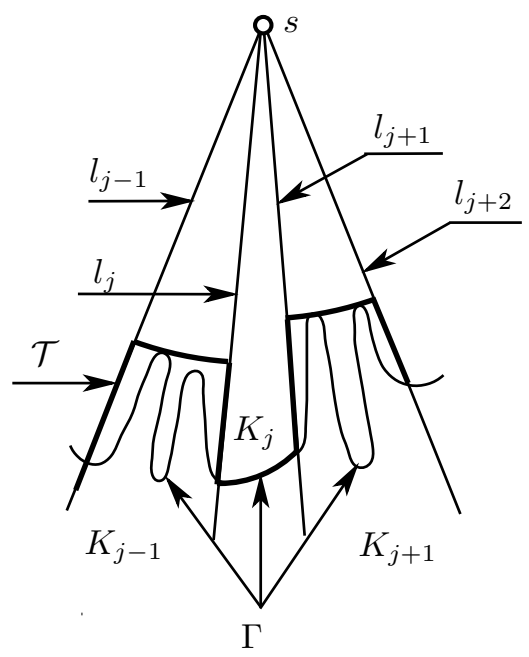

Рис. 4 
из точек $a, b$ есть точка одностороннего локального минимума функции $\rho_{\Gamma}(\varphi)$ на конусе $K$. Обозначим

$$
\Delta=\Delta_{x}=\left[\varphi_{\rho^{*}}, \varphi^{\rho^{*}}\right] .
$$

Если точка максимума $x$ не является точкой строгого локального максимума, то указанный отрезок $\Delta$ порождается и другими точками $y$, для которых $\Delta=\Delta_{y}$. Пусть $C_{\rho}^{a b}$ - дуга окружности $C_{\rho}$ между точками $a, b$, пересекающая отрезок $[s, x]$, и $G_{\rho}^{a b}$ - часть графика gr $\rho(\cdot)$ между точками $a, b$, содержащая $x$.

Требуется решить следующую задачу поиска непрерывной функции $\gamma=\gamma(\varphi)$ на $\Delta$, доставляющую минимум

$$
J_{\Delta_{x}}=\min _{\gamma}\left\{\int_{\Delta} \widetilde{F}(\gamma(\varphi)) d \varphi: \gamma(\varphi) \leq \rho_{\Gamma}(\varphi) \text { для } \varphi \in \Delta, \gamma\left(\varphi_{\rho^{*}}\right)=\gamma\left(\varphi^{\rho^{*}}\right)=\rho^{*}\right\} .
$$

Ясно, что график функции $\widehat{\gamma}=\widehat{\gamma}(\varphi)$, являющейся решением задачи (6) расположен между кривыми $C_{\rho^{*}}^{a b}, G_{\rho^{*}}^{a b}$. Заметим, что на тех отрезках $\left[\varphi_{1}, \varphi_{2}\right] \subset \Delta, \varphi_{1}<\varphi_{2}$, где $\widehat{\gamma}(\varphi)<\rho_{\Gamma}(\varphi)$, функция $\widehat{\gamma}$ является выпуклой вниз. В самом деле, если это не так, то найдутся $\varphi^{\prime}, \varphi^{\prime \prime}$, $\varphi_{1} \leq \varphi^{\prime}<\varphi^{\prime \prime}<\varphi_{2}$ такие, что график функции $\widehat{\gamma}(\varphi), \varphi^{\prime}<\varphi<\varphi^{\prime \prime}$, расположен между $s$ и прямолинейным отрезком, соединяющим точку $\left(\widehat{\gamma}\left(\varphi^{\prime}\right), \varphi^{\prime}\right),\left(\widehat{\gamma}\left(\varphi^{\prime \prime}\right), \varphi^{\prime \prime}\right)$. Зеркально отобразив этот график относительно указанного прямолинейного отрезка, легко, воспользовавшись свойством (2), построить траекторию с меньшей величиной облучения в сравнении с $(\widehat{\gamma}(\varphi), \varphi)$.

Для каждой точки локального максимума $x$ (точнее, для каждого отрезка, состоящего из точек локального минимума) решим задачу (6) на $\Delta_{x}$. Справедлива

Теорема. Пусть функиия $\rho_{\Gamma}(\varphi)$ кусочно монотонна. Экстремальная траектория задачи (5) задается функиией $\widehat{\rho}(\varphi)=\widehat{\rho}(\varphi, s)(0 \leq \varphi \leq \Phi)$, которая совпадает с функцией $\widehat{\gamma}(\varphi)(\varphi \in \Delta)$ на каждом отрезке $\Delta=\Delta_{x}$, а на множестве $\Phi \backslash \bigcup_{x} \Delta_{x}$ совпадает с $\rho_{\Gamma}(\varphi)$.

Для д о к а з а т е л ь с в а теоремы достаточно заметить, что ввиду монотонности функций $F$, на множестве $\Phi \backslash \bigcup_{x} \Delta_{x}$ экстремальная траектория совпадает с $\rho_{\Gamma}(\varphi)$, а вне этого множества она построена оптимально и совпадает с $\widehat{\gamma}(\varphi)$.

Таким образом, исходная задача (5) сводится к серии экстремальных задач вида (6).

Для числа $\rho, \rho^{*} \leq \rho \leq \bar{\rho}$ определим функцию

$$
\delta_{\rho}(\varphi)=\min \left\{\rho_{\Gamma}(\varphi), \rho\right\} \text { для } \varphi \in \Delta_{x} .
$$

Представляет интерес более простая в сравнении с (6) задача поиска минимума на более узком классе функций:

$$
\bar{J}_{\Delta}=\min _{\rho^{*} \leq \rho \leq \bar{\rho}} \int_{\Delta} \tilde{F}\left(\delta_{\rho}(\varphi)\right) d \varphi
$$

решение данной задачи аппроксимирует функцию $\widehat{\gamma}(\cdot)$.

Отметим еще один способ построения траектории $\mathcal{T}$, близкой к оптимальной. Разобьем конус $K(s)$ на частичные конусы $K_{i}$, ограниченные лучами $l_{j}, l_{j+1}$, где $l_{j}=l_{\varphi_{j}}\left(0=\varphi_{0}<\right.$ $\left.\varphi_{1}<\cdots<\varphi_{m}=\Phi\right), \Delta_{j}=\Delta_{j} \varphi=\left[\varphi_{j}, \varphi_{j+1}\right)$. В простейшем случае разбиение является равномерным либо оно определяется свойствами границы $Г$. Построим траекторию $\mathcal{T}$, основываясь на функции $t(\varphi)$, постоянной на некоторых частичных интервалах $\Delta_{j}$ с большой вариацией функции $\rho_{\Gamma}(\varphi)$. Не нарушая ограничения $\rho(\varphi) \leq \rho_{\Gamma}(\varphi)$, ввиду отмеченного выше (см. (4)) свойства монотонности по $\lambda$ величины $\int \widetilde{F}(\lambda \rho(\varphi)) d \varphi$ естественно на этих интервалах взять кусочно постоянную функцию

$$
t(\varphi)=\rho_{j}=\min \left\{\rho_{\Gamma}(\alpha): \alpha \in \Delta_{j} \varphi\right\} \text { при } \varphi \in \Delta_{j} \varphi .
$$

Траектория $\mathcal{T} \subset Y$ движения объекта на выбранных интервалах строится пополнением графика функции $t(\varphi)$ в каждой точке разрыва $\varphi_{j}$ отрезком $\left[a_{j}, b_{j}\right]$ луча $l_{j}$, где $a_{j}=\left(\rho_{j}, \varphi_{j}\right), b_{j}=$ $\left(\rho_{j-1}, \varphi_{j}\right)$. На оставшихся частичных интервалах $\mathcal{T}$ совпадает с $\rho_{\Gamma}(\varphi)$ (рис. 4$)$. 


\section{3. Случай двух источников в $\mathbb{R}^{2}$}

Точки $t_{*}, t^{*}$ разбивают границу $\partial Y$ на две части $\Gamma_{1}, \Gamma_{2}$. Далее считаем $s_{i} \in \Gamma_{i}(i=1,2)$, т. е. источники расположены на противоположных частях границы $\partial Y$ и

$$
F_{1}=F_{2} \stackrel{\text { def }}{=} F, \quad\left[s_{1}, s_{2}\right] \cap\left(\left(\stackrel{\circ}{K}_{Y}\left(s_{1}\right) \cup \stackrel{\circ}{K}_{Y}\left(s_{2}\right)\right)=\varnothing\right.
$$

(см. рис. 5). Обозначим $K_{Y}\left(s_{1}\right) \cap K_{Y}\left(s_{2}\right)=\mathcal{D}$, а через $l_{i}, l_{i}^{\prime}$ - "левый" и "правый" лучи, ограничивающие конус $K\left(s_{i}\right), i=1,2$. Рассмотрим возможные варианты расположения источников $s_{1}, s_{2}$ и способы построения оптимальных траекторий. Пусть $L-$ прямая, ортогональная отрезку $\left[s_{1}, s_{2}\right]$, содержащая точку $\left(s_{1}+s_{2}\right) / 2$.

Если $\mathcal{D} \cap L=\varnothing$ и множества $\mathcal{D}, K_{Y}\left(s_{1}\right) \cap \Gamma_{1}$, к примеру, лежат по одну сторону от $L$, то, как можно видеть, оптимальной является траектория $\mathcal{T}_{2,1}$, пересекающая конус $K_{Y}\left(s_{2}\right)$ по отрезку $A_{2}=l_{1} \cap K_{V}\left(s_{2}\right)$, а конус $K_{Y}\left(s_{1}\right)$ - по графику функции $\rho_{\Gamma_{2}}: G_{1} \stackrel{\text { def }}{=}\left(\operatorname{gr} \rho_{2}(\cdot)\right) \cap K_{Y}\left(s_{1}\right)$ (на рис. 6) эти участки траектории отмечены жирной пунктирной линией).

Теперь предположим, что $\stackrel{\circ}{\mathcal{D}} \cap L \neq \varnothing$.

Среди траекторий, не пересекающих множество $\stackrel{\circ}{\mathcal{D}}$, имеется две траектории, претендующие на оптимальность; это $\mathcal{T}_{1,2}, \mathcal{T}_{2,1}$, пересекающие конусы $K_{Y}\left(s_{i}\right)$ поочередно. Вторая определена выше, а траектория $\mathcal{T}_{1,2}$ пересекается с $K_{Y}\left(s_{1}\right)$ - по отрезку $A_{1}=l_{2} \cap K_{Y}\left(s_{1}\right)$ и с конусом $K_{Y}\left(s_{2}\right)$ - по графику функции $\rho_{\Gamma_{1}}: G_{2} \stackrel{\text { def }}{=}(\operatorname{gr} \rho(\cdot)) \cap K_{Y}\left(s_{2}\right)$ (рис. 7). Этим траекториям соответствуют величины облучения

$$
J_{1,2}=\int_{A_{1}} F\left(s_{1}-x\right) d x+\int_{G_{2}} F\left(s_{2}-x\right) d x, \quad J_{2,1}=\int_{A_{2}} F\left(s_{2}-x\right) d x+\int_{G_{1}} F\left(s_{1}-x\right) d x .
$$

Рассмотрим траектории, пересекающие множество $\stackrel{\circ}{\mathcal{D}}$. Все они содержат отрезок $\left[a, a^{\prime}\right]=$ $L \cap \mathcal{D}$, обозначенный с учетом направления движения от $t_{*} \mathrm{~K} t^{*}$. В самом деле, дифференцируя по $\alpha$ функцию $F\left(s_{1}-x-\alpha\left(s_{1}-s_{2}\right)\right)+F\left(s_{2}-x-\alpha\left(s_{1}-s_{2}\right)\right)$, убеждаемся, что минимум по $\alpha$ этой функции достигается при $\alpha=0$ для всех $x \in\left[a, a^{\prime}\right]$. Поиск оптимальной траектории вне множества $\mathcal{D}$ может осуществляться посредством решения следующей задачи.

Пусть $l$ - луч из $K(s), \eta$ - отрезок из $l, c$ - точка из $K_{Y}(s) \backslash l, \mathbb{T}(x, c)-$ совокупность траекторий вида $\mathcal{T}_{x}=\{t(\tau): 0 \leq \tau \leq 1, t(0)=x, t(1)=c\} \subset K_{Y}(s), x \in \eta$. Требуется найти величину

$$
J(\eta, c)=\inf _{x \in \eta} \inf \left\{\int_{0}^{1} F_{s}(t(\tau)) d \tau: \mathcal{T} \in \mathbb{T}(x, c)\right\} .
$$

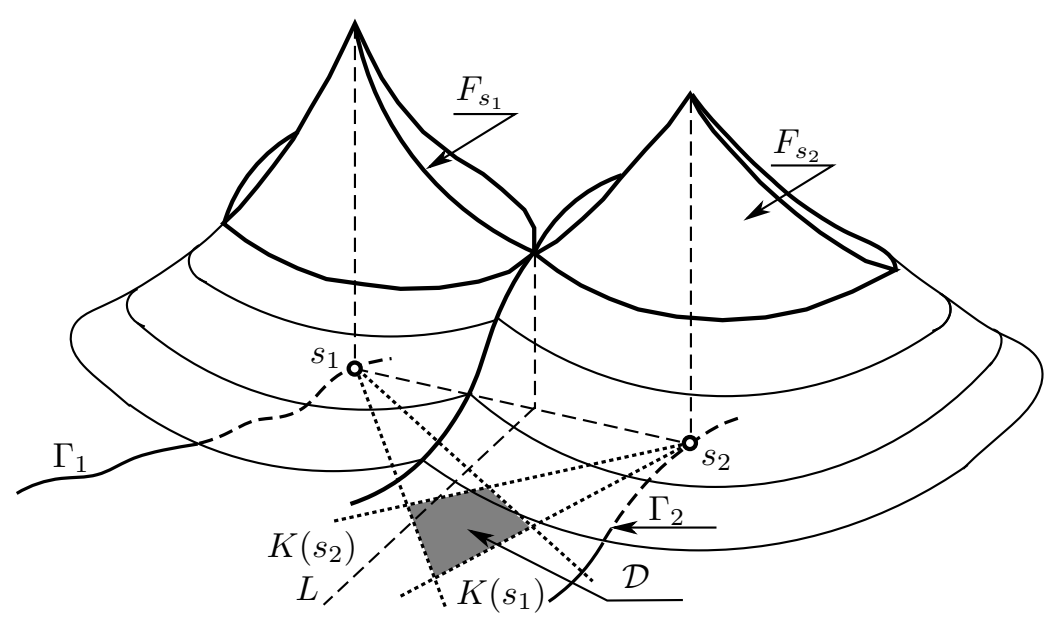

Рис. 5 


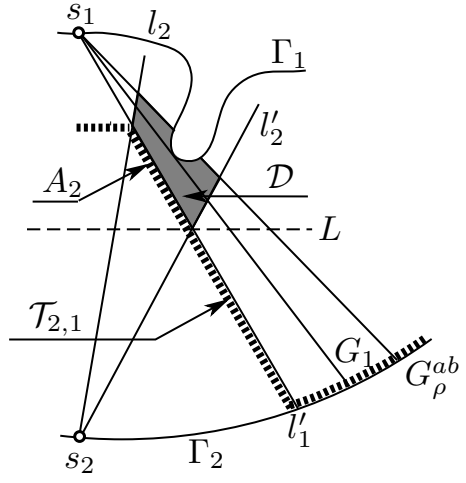

Рис. 6

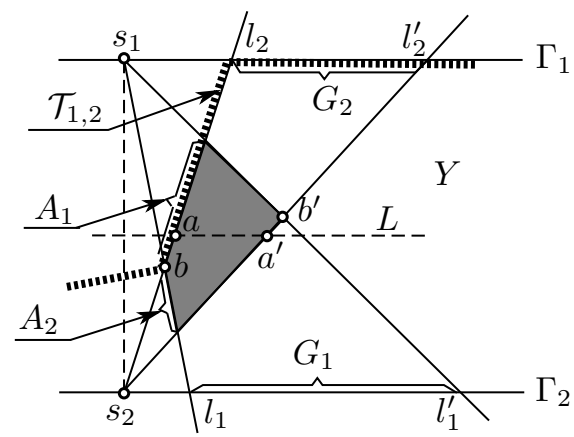

Рис. 7

Обозначим $\left[q, q^{\prime}\right]=L \cap\left(K_{Y}\left(s_{1}\right) \cup K_{Y}\left(s_{2}\right)\right), b=l_{1} \cap l_{2}, q_{1,2}^{\prime}=l_{1}^{\prime} \cap \Gamma_{2}, q_{2,1}^{\prime}=l_{2}^{\prime} \cap \Gamma_{1}$. Легко убедиться, что для оптимального подхода из точки $t_{*}$ в точку $a$ объект должен пересечь отрезок $[q, b]$, используя решение задачи $J([q, b], a)$, а для выхода из точки $a^{\prime}$ в сторону точки $t^{*}$ он должен пересечь отрезок $\left[q^{\prime}, q_{1,2}^{\prime}\right]$, если $q^{\prime} \in l_{1}^{\prime}$ (рис. $8 a$ ), и пересечь отрезок $\left[q^{\prime}, q_{2,1}^{\prime}\right]$, если $q^{\prime} \in l_{2}^{\prime}$ (рис. 8б).

Далее понадобится

Лемма. Пусть $s=0$, точки $v, w$ принадлежат конусу $K_{Y}(s), w \notin l=\{\alpha v, \alpha>0\}$,

$$
J(\alpha)=\int_{0}^{1} F_{s}(\lambda \alpha v+(1-\lambda) w) d \lambda, \quad \alpha>0 .
$$

Тогда функиия $J(\alpha)$ является убъвающей.

Д о к а з а т е л ь с т в о. В самом деле,

$$
\begin{aligned}
\frac{1}{\Delta \alpha}[J(\alpha+\Delta \alpha)-J(\alpha)] & =\frac{1}{\Delta \alpha} \int_{0}^{1}[F(\lambda(\alpha+\Delta \alpha) v+(1-\lambda) w)-F(\lambda \alpha v+(1-\lambda) w)] d \lambda \\
& \underset{\Delta \alpha \rightarrow 0}{\longrightarrow} \int_{0}^{1} F_{\alpha}^{\prime}(\lambda \alpha v+(1-\lambda) w) \lambda d \lambda<0 .
\end{aligned}
$$
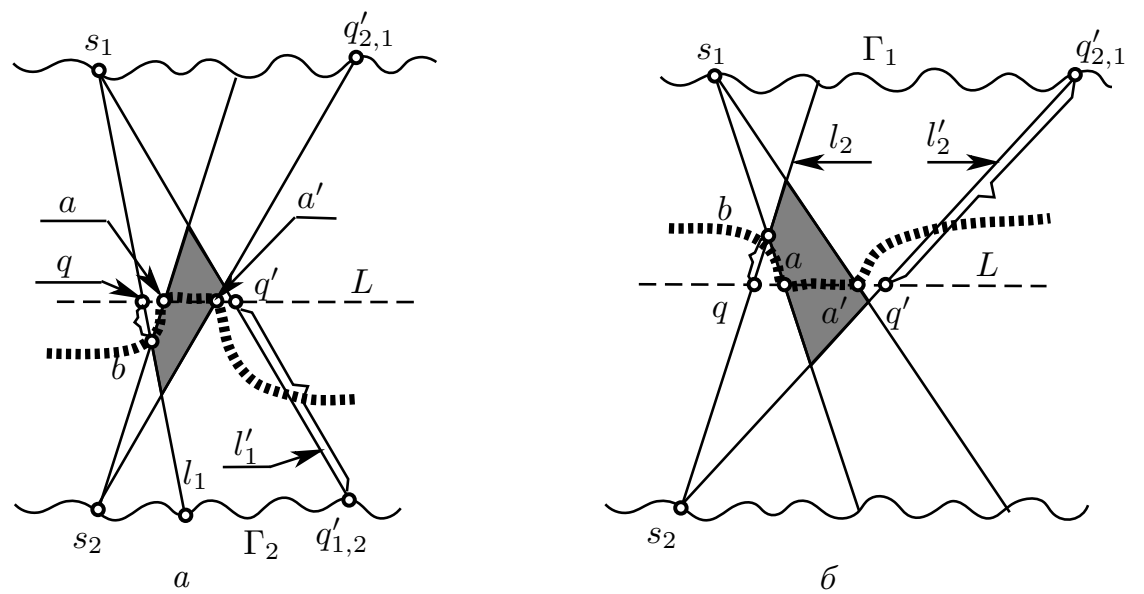

Рис. 8 


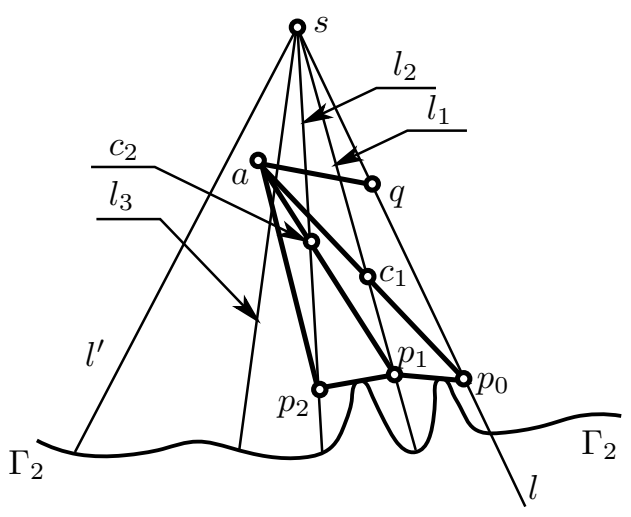

Рис. 9

Используя лемму, приходим к выводу, что одна искомая часть траектории до точки $a$ совпадает с отрезком $[a, b]$ (см. рис. $8 a$ и 8 б). Для построения другой части траектории от точки $a^{\prime}$ до $t^{*}$ используем следующий алгоритм поиска приближенного решения задачи $J\left(\left[q, p_{0}\right], a\right)(8)$, где $a \in \stackrel{\circ}{K}(s), l^{\prime}$ и $l$ - граничные лучи с вершиной $s$ конуса $K(s), p_{o} \in l, q \in(s, p)$. Будем использовать обозначение $\int_{a}^{b}=\int_{a}^{b} F(x) d x$, где $[a, b]$ - прямолинейный отрезок.

Зафиксируем конечный набор лучей $l_{i} \in K(s)$ с вершиной $s$, пересекающих интервал $(a, q)$ в порядке от точки $q$ к точке $a$. Пусть $p_{1} \in l_{1}$ - наиболее удаленная от $s$ точка такая, что отрезок $\left[p_{1}, p_{0}\right]$ не пересекается внутренностью множества $X \backslash Y$. Обозначим $c_{i}=l_{i} \cap\left[a, p_{i-1}\right]$. Применяя лемму (см. рис. 9),

$$
\text { при } w=a, \text { получим неравенство } \int_{a}^{q}>\int_{a}^{p_{0}} ;
$$

$$
\left.\begin{array}{c}
\text { при } w=p_{0}-\text { неравенство } \int_{c_{1}}^{p_{0}}>\int_{p_{0}}^{p_{1}} ; \\
\text { при } \quad w=a-\text { неравенство } \int_{a}^{c_{1}}>\int_{a}^{p_{1}}
\end{array}\right\} \Rightarrow \int_{a}^{p_{0}}>\int_{a}^{p_{1}}+\int_{p_{1}}^{p_{0}} .
$$

Пусть $p_{2} \in l_{2}$ - наиболее удаленная от $s$ точка, такая что отрезок $\left[p_{1}, p_{2}\right]$ не пересекается с внутренностью множества $X \backslash Y$. Применяя лемму,

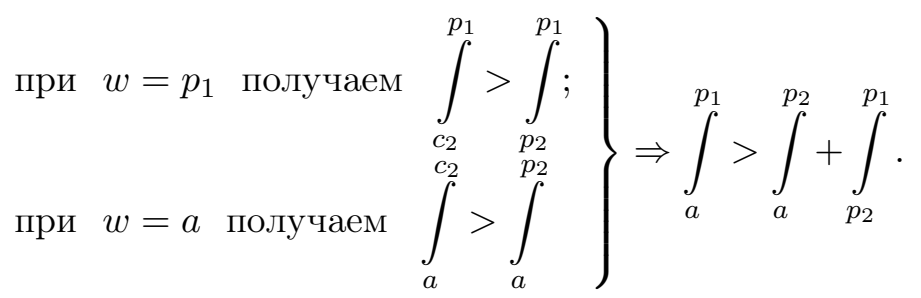

Из (9)-(11) следует, что

$$
\int_{a}^{q}>\int_{a}^{p_{0}}>\int_{a}^{p_{1}}+\int_{p_{1}}^{p_{0}}>\int_{a}^{p_{2}}+\int_{p_{2}}^{p_{1}}+\int_{p_{1}}^{p_{0}} .
$$

Таким образом, построены четыре траектории: $[a, q],\left[a, p_{0}\right],\left[a, p_{1}\right] \cup\left[p_{1}, p_{0}\right],\left[a_{0}, p_{2}\right] \cup\left[p_{2}, p_{1}\right] \cup$ $\left[p_{1}, p_{0}\right]-$ с убывающей величиной совокупного облучения, которые соединяют точку $a$ с отрезком $\left[q, p_{0}\right]$. Процесс построения траекторий можно продолжить, используя все лучи из 
заданного набора $\left\{l_{i}\right\}$. Увеличивая набор $\left\{l_{i}\right\}$, мы построим траекторию, для которой участок $\left[p_{0}, p_{1}\right] \cup\left[p_{1}, p_{2}\right] \ldots\left[p_{k}, p_{k+1}\right]$ лучше аппроксимирует участок границы $\Gamma_{2}$. Более того, можно задать бесконечный набор лучей в $l_{i}$, приближающихся к точке, и построить "сходящуюся" последовательность траекторий.

Таким образом, при наличии пары источников $s_{1}, s_{2}$, имеющих непустое пересечение $\stackrel{\circ}{K}_{Y}\left(s_{1}\right) \cap \stackrel{\circ}{K}_{Y}\left(s_{2}\right)$, для поиска оптимальной траектории следует определить, к какому из рассмотренных случаев относится взаимоположение конусов $K_{Y}\left(s_{i}\right)$.

Если источники лежат на одной части границы $\partial Y$, например на $\Gamma_{1}$ и $\stackrel{\circ}{K}_{Y}\left(s_{1}\right) \cap \stackrel{\circ}{K} Y\left(s_{2}\right) \neq \varnothing$, то в силе остаются рассуждения, проведенные в случае единственного облучателя.

\section{4. Приближенное построение оптимальных траекторий в $\mathbb{R}^{3}$}

Рассмотрим случай единственного излучателя $s$. Предполагаем, что граница $\partial Y$ не препятствует излучению, т. е. не образует теней, $s \notin Y$, любая траектория $\mathcal{T} \in \mathbb{T}$ пересекает внутренность усеченного конуса $K_{Y}(s)=K(s) \cap Y$ и при этом $K_{Y}$ - ближайшая к $s$ связная часть коридора $Y$, обладающая указанным свойством. Будем исходить из того, что раствор конуса $K(s)$ меньше $\pi$. Построим при выбранном $m$ два однопараметрических семейства плоскостей

$$
\left\{P^{i}\right\},\left\{Q^{j}\right\}, \quad i, j \in\{1,2, \ldots, m\} .
$$

Пусть $\mathcal{L}$ и $\mathcal{R}$ - левая и, соответственно, правая стороны поверхности $Y \cap \partial K(s)$ (в том смысле, что любая траектория из $\mathbb{T}$ входит в усеченный конус $K_{Y}(s)$ через $\mathcal{L}$ и выходит из него через $\mathcal{R}), p_{l}$ и $p_{r}$ - точки из пересечения коридора $Y$ и поверхностей $\mathcal{L}, \mathcal{R}$ соответственно, $P_{l}, P_{r}$ - касательные плоскости к $\mathcal{L}$ и $\mathcal{R}$ в точках $p_{l}$ и $p_{r}$. Ясно, что $s \in P_{l} \cap P_{r}$. Пусть $Q_{I}, Q_{I I}-$ плоскости, содержащие точку $s$, опорные $K_{Y}(s)$ с разных сторон коридора $Y$. Определим

$$
P^{i}=\frac{1}{i} P_{l}+\left(1-\frac{1}{i}\right) P_{r}, \quad Q^{j}=\frac{1}{j} Q_{I}+\left(1-\frac{1}{j}\right) Q_{I I}, \quad i, j \in\{1,2, \ldots, m\}
$$

при заданном $m$. Плоскости $P^{i}, Q^{j}$ разбивают конус $K(s)$ на частичные конусы $K_{i j}$, ограниченные плоскостями $P^{i}, P^{i+1}, Q^{j}, Q^{j+1}$. Пусть для луча $l \subset K_{i j}$ точка $z(l) \in l \cap K_{Y}(s)$ наиболее удалена от $s$. Среди таких лучей найдем луч $l=l_{i j}$, для которого величина $\left\|s-z\left(l_{i j}\right)\right\|$ наибольшая. Обозначим $z_{i j}=z\left(l_{i j}\right)$. Построим граф, вершинами которого являются точки $z_{i j}$, ребрами - отрезки, соединяющие точки $z_{i j}, z_{i^{\prime} j^{\prime}}$ из соседних частичных конусов, т. е. конусов, имеющих общее ребро или общую грань, содержащие точку $s$. Для определения веса ребра $\left[z_{i j}, z_{i^{\prime} j^{\prime}}\right]$ найдем расстояние $\rho_{z}$ от $s$ до границы $\partial Y$ вдоль луча $\{s+\alpha(z-s), \alpha>0\}$, где $z=\lambda z_{i j}+(1-\lambda) z_{i^{\prime} j^{\prime}}$. Вес ребра определим в виде

$$
\int_{0}^{1} \widetilde{F}\left(\rho_{z}\left(\lambda z_{i j}+(1-\lambda) z_{i^{\prime} j^{\prime}}\right)\right) d \lambda .
$$

Пусть $Z_{l}$ - множество вершин $z_{i j}$ графа, которые соответствуют частичным конусам $K_{i j}$, пересекающимся с левой стороной $\mathcal{L}$ множества $Y \cap \partial K(s)$, аналогично определяется множество вершин $Z_{r}$. Задача построения траектории, аппроксимирующей оптимальную, сводится к поиску пути на графе, соединяющего множества $Z_{l}$ и $Z_{r}$ и имеющего наименьшую сумму весов его ребер.

Рассмотрим случай двух излучателей $s_{1}, s_{2}$ в $\mathbb{R}^{3}$. Далее предполагаем, что $F_{s_{1}}=F_{s_{2}}$, множество $\mathcal{D}=\stackrel{\circ}{K} Y\left(s_{1}\right) \cap \stackrel{\circ}{K} Y\left(s_{2}\right)$ не пусто; кроме того, можно считать, что $\left[s_{1}, s_{2}\right] \cap \stackrel{\circ}{\mathcal{D}}=\varnothing$, поскольку излучатели $s_{1}, s_{2}$ не должны облучать друг друга. Здесь, как и ранее, используются обозначения $\mathcal{L}_{i}=\mathcal{L} K_{Y}\left(s_{i}\right), \mathcal{R}_{i}=\mathcal{R} K_{Y}\left(s_{i}\right)$ - левая и правая стороны усеченного конуса $K_{Y}\left(s_{i}\right)$, а часть коридора $Y$, расположенная, например, справа от $\mathcal{R}_{2}$ и слева от $\mathcal{L}_{1}$, называемая боксом, 


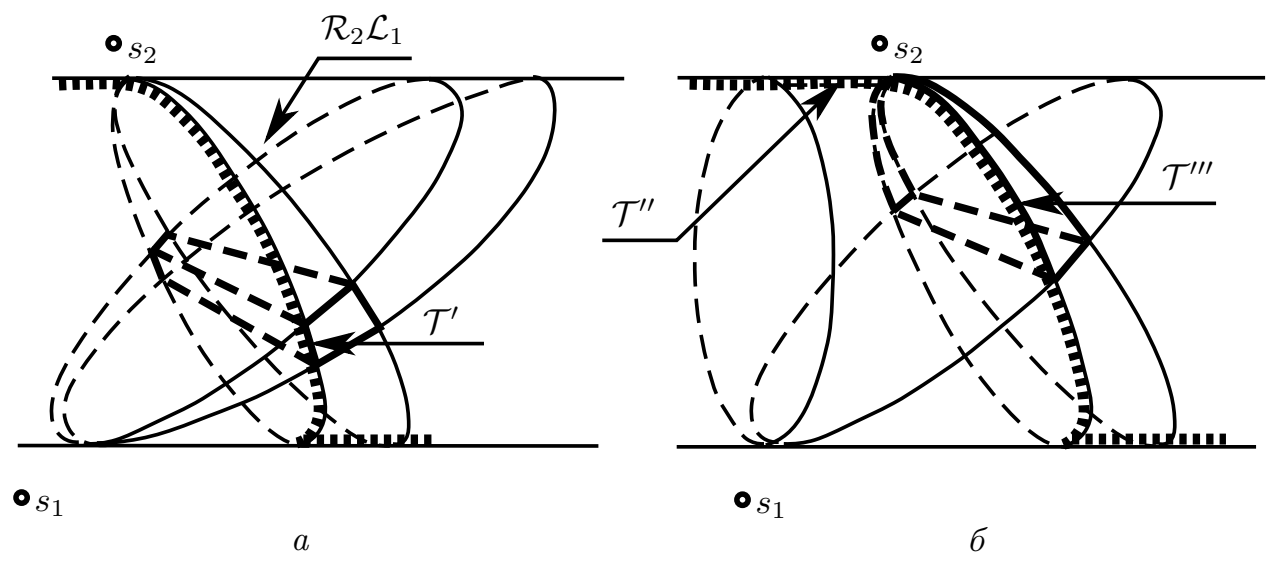

Рис. 10

обозначается через $\mathcal{R}_{2} \mathcal{L}_{1}$. Возможные положения конусов $K\left(s_{1}\right), K\left(s_{2}\right)$ изображены на рис. 10 . Ребра границы множества $\mathcal{D}$ отмечены жирными непрерывной и пунктирной линиями. Имеем $s_{1} \notin K\left(s_{2}\right), s_{2} \notin K\left(s_{1}\right)$ и $\mathcal{D}$ является пересечением с $Y$ четырехгранника с выпуклыми коническими гранями в случае $a$; в случае $\sigma s_{2} \in K\left(s_{1}\right)$ и $\mathcal{D}=Y \cap K\left(s_{1}\right) \cap K\left(s_{2}\right)$.

Для определенности будем считать, что отрезок $\left[s_{1}, s_{2}\right]$ расположен "слева" от множества $\mathcal{D}$. Обозначим через $L$ плоскость, ортогональную этому отрезку и содержащую точку $\left(s_{1}+s_{2}\right) / 2$.

Сперва предположим, что $L \cap \mathcal{D}=\varnothing$. В этом случае оптимальная траектория $\mathcal{T}$ не пересекается с $\mathcal{D}$, поскольку если $t \in \mathcal{T} \cap \mathcal{D}$ и $\rho\left(s_{1}, t\right) \neq \rho\left(s_{2}, t\right)$, то точку $t$ можно сместить в точку $t^{\prime} \in \mathcal{D}$ такую, что

$$
\rho\left(s_{1}, t\right)+\rho\left(s_{2}, t\right)<\rho\left(s_{1}, t^{\prime}\right)+\rho\left(s_{2}, t^{\prime}\right) .
$$

В самом деле, когда $\rho\left(s_{1}, t\right)>\rho\left(s_{2}, t\right)$, можно взять $t^{\prime}=s_{2}+\varepsilon\left(t-s_{2}\right)$ при малом $\varepsilon>0$. Отсюда $\mathcal{T}$ пересекает последовательно конусы $K\left(s_{i}\right)$, например сперва $K\left(s_{1}\right)$ и затем $K\left(s_{2}\right)$ (случай другой очередности рассматривается аналогично). Итак, задача состоит в поиске траектории $\mathcal{T}$, оптимально пересекающей $K\left(s_{1}\right)$ при переходе из бокса $\mathcal{L}_{1} \mathcal{L}_{2}$ в бокс $\mathcal{R}_{1} \mathcal{L}_{2}$; далее надо найти траекторию, оптимально пересекающую $K\left(s_{2}\right)$ при переходе из бокса $\mathcal{R}_{1} \mathcal{L}_{2}$ в бокс $\mathcal{R}_{1} \mathcal{R}_{2}$. Оптимальность означает минимизацию совокупного облучения объекта на каждом участке $\delta \mathcal{T}_{i}=\mathcal{T} \cap K\left(s_{i}\right), i=1,2$. Поведение оптимальной траектории на коротком участке можно отслеживать по точкам пересечения с плоскостями специально подобранного однопараметрического семейства, подобного семейству $P_{\lambda}=\lambda P_{l}+(1-\lambda) P_{r}$ (см. (12)). Это семейство должно рассекать часть пространства и траекторию, которая заведомо содержит участок $\delta \mathcal{T}_{i}$ траектории. Учитывая выпуклость конусов $K\left(s_{i}\right)(i=1,2)$ и подбирая подходящее однопараметрическое семейство плоскостей, убеждаемся, что

- в случае $а$ наиболее далекая от $s_{1}$ траектория пересечения конуса $K\left(s_{1}\right)$, обозначим ее как $\mathcal{T}^{\prime}$, принадлежит множеству $Y \cap K\left(s_{1}\right) \cap \mathcal{L}_{2}$,

- в случае $\sigma$ наиболее удаленная от $s_{1}$ траектория составлена из двух частей $\mathcal{T}^{\prime \prime}, \mathcal{T}^{\prime \prime \prime}$, первая из которых $\mathcal{T}^{\prime \prime}$ принадлежит $\partial Y$ и соединяет $\mathcal{L}_{1}$ и $\mathcal{L}_{2}$, а вторая $\mathcal{T}^{\prime \prime \prime}$ принадлежит множеству $Y \cap K\left(s_{1}\right) \cap \mathcal{L}_{2}$.

В обоих случаях осталось построить траекторию $\mathcal{T}_{2}$, оптимально пересекающую конус $K\left(s_{2}\right)$. Как отмечалось выше, наиболее удаленная от $s_{i}$ кривая не всегда является оптимальной; необходимо учитывать ее длину. Для поиска траектории, близкой к оптимальной, можно использовать способы, предложенные в предыдущих разделах: при построении $\mathcal{T}^{\prime}$ и $\mathcal{T}^{\prime \prime \prime}$ - методы, изложенные в разделе, в котором исследуется задача в $\mathbb{R}^{2}$, а для поиска $\mathcal{T}^{\prime \prime}-$ алгоритм поиска траектории в $\mathbb{R}^{3}$ в случае единственного излучателя. На рис. 10 одна из оптимальных траекторий изображена точечной линией. 
Пусть $L_{\mathcal{D}} \stackrel{\text { def }}{=} L \cap \mathcal{D}$. Точки $x \in \mathcal{D}$ получают облучение величины $2 F\left(\left\|s_{1}-x\right\|\right)$ из виртуального источника $\left(s_{1}+s_{2}\right) / 2$. Множество $\mathcal{D}$ есть пересечение $Y$ с выпуклым множеством, граница которого состоит в случае $a$ из фрагментов четырех (трех в случае б) конических поверхностей

$$
\mathcal{L}_{1} \cap K_{2}, \quad \mathcal{R}_{1} \cap K_{2}, \quad \mathcal{L}_{2} \cap K_{1}, \quad \mathcal{R}_{2} \cap K_{1},
$$

где $K_{i}=K\left(s_{i}\right)$. В простейшем случае, когда плоскость $L$ пересекает ребра $\widetilde{l}=\left(\mathcal{L}_{1} \cap \mathcal{L}_{2}\right) \cap Y, \widetilde{r}=$ $\left(\mathcal{R}_{1} \cap \mathcal{R}_{2}\right) \cap Y$, задача сводится к поиску оптимальной плоской кривой из $L_{\mathcal{D}}$, соединяющей точки пересечения $L$ с указанными ребрами, которую можно построить с помощью приведенной выше леммы. Оптимальная траектория вне $\mathcal{D}$ принадлежит боксам $\mathcal{L}_{1} \mathcal{L}_{2}$ и $\mathcal{R}_{1} \mathcal{R}_{2}$, которые лежат вне зоны облучения.

В общем случае найдем расстояния

$$
\min \left\{\|x-d\|: x \in \widetilde{l}, d \in L_{\mathcal{D}}\right\}, \quad \min \left\{\|y-d\|: y \in \widetilde{r}, d \in L_{\mathcal{D}}\right\}
$$

и пары точек $x_{l} \in \widetilde{l}, d_{l} \in L_{\mathcal{D}}, y_{r} \in \widetilde{r}, d_{r} \in L_{\mathcal{D}}$, реализующие эти нижние грани. Для решения задачи достаточно построить оптимальную траекторию в $L_{\mathcal{D}}$, соединяющую точки $d_{l}, d_{r}$, и две траектории в $\overline{\mathcal{D}}$ : одна соединяет точки $x_{l}, d_{l}$, другая - точки $y_{r}, d_{r}$.

Приведенные способы позволяют в разных случаях расположения конусов $K(s)$ строить либо оптимальную, либо близкую к оптимальной траекторию.

\section{СПИСОК ЛИТЕРАТУРЫ}

1. Лю В. Методы планирования пути в среде с препятствиями (обзор) // Математика и мат. моделирование. 2018. № 1. С. 15-58. doi: 10.24108/mathm.0118.0000098.

2. Арутюнов А.В., Магарил-Ильяев Г.Г., Тихомиров В.М. Принцип максимума Понтрягина. Доказательство и приложения. Москва: Факториал Пресс, 2006. 144 с.

3. Коробкин В.В., Сесекин А.Н., Ташлыков О.Л., Ченцов А.Г. Методы маршрутизации и их приложения в задачах повышения безопасности и эффективности атомных станций. Москва: Новые технологии, 2012. $234 \mathrm{c}$.

4. Chentsov A.G., Grigoryev A.M., Chentsov A.A. Optimization "In windows" for routing problems with constraints // Mathematical Optimization Theory and Operations Research - 18th International Conference / eds. I. Bykadorov, V. Strusevich, T. Tchemisova (MOTOR 2019): Revised Selected Papers. N Y, Berlin: Springer Verlag, 2019. pp. 470-485. (Communications in Computer and Information Science; vol. 1090 CCIS). doi: 10.1007/978-3-030-33394-2_36.

Поступила 25.12.2019

После доработки 23.01.2020

Бердышев Виталий Иванович

Принята к публикации 27.01.2020

академик РАН

научный руководитель

Институт математики и механики им. Н. Н. Красовского УрО РАН

г.Екатеринбург

e-mail: bvi@imm.uran.ru

Костоусов Виктор Борисович

канд. физ.-мат. наук

зав. отд.

Институт математики и механики им. Н.Н. Красовского УрО РАН, г.Екатеринбург e-mail: vkost@imm.uran.ru 


\section{REFERENCES}

1. Liu W. Path planning methods in an environment with obstacles (A review). Matematika $i$ Mat. Modelirovanie, 2018, no. 1, pp. 15-58 (in Russian). doi: 10.24108/mathm.0118.0000098.

2. Arutyunov A.V., Magaril-Il'yaev G.G., Tikhomirov V.M. Princip maximuma Pontryagina. Dokazatel'stvo i prilozheniya [The Pontryagin maximum principle. Proof and applications]. Moscow: Faktorial Press Publ., 2006, 144 p. ISBN: 5886880828. (in Russian).

3. Korobkin V.V., Sesekin A.N., Tashlyikov O.L., Chentsov A.G. Metody marshrutizacii i ih prilozheniya $v$ zadachah povyisheniya bezopasnosti $i$ effektivnosty atomnykh stancii [Methods of routing and their appendix in problems of increase of efficiency and safety of operation of nuclear power plants]. Moscow: Novyie Tekhnologii Publ., 2012, 234 p. (in Russian).

4. Chentsov A.G., Grigoryev A.M., Chentsov A.A. Optimization "In windows" for routing problems with constraints. Mathematical Optimization Theory and Operations Research - 18th International Conference / eds. I. Bykadorov, V. Strusevich, T. Tchemisova (MOTOR 2019): Revised Selected Papers, Communications in Computer and Information Science; vol. 1090 CCIS, N Y, Berlin: Springer Verlag, 2019. P. 470-485. doi: 10.1007/978-3-030-33394-2_36.

Received December 25, 2019

Revised January 23, 2020

Accepted January 27, 2020

Vitalii Ivanovich Berdyshev, RAS Academician, Krasovskii Institute of Mathematics and Mechanics Ural Branch of the Russian Academy of Sciences, Yekaterinburg, 620108 Russia, e-mail: bvi@imm.uran.ru .

Viktor Borisovich Kostousov, Cand. Sci. (Phys.-Math.), Krasovskii Institute of Mathematics and Mechanics Ural Branch of the Russian Academy of Sciences, Yekaterinburg 620108 Russia, e-mail: vkost@imm.uran.ru .

Cite this article as: V.I. Berdyshev, V.B. Kostousov. A trajectory minimizing the exposure of a moving objects, Trudy Instituta Matematiki i Mekhaniki URO RAN, 2020, vol. 26, no. 1, pp. 27-38 . 